We wish to acknowledge the help of Mr. J. M. Brudenell, Dr. W. G. Oakley, Dr. D. A. Pyke, Mr. G. D. Pinker, Dr. C. A. Young, Mr. R. L. Lloyd-Jones, and Dr. J. D. N. Nabarro for allowing us to study their cases; Professor V. Wynn, in whose laboratory the biochemical estimations were performed, for his support and encouragement; Miss Cynthia Knight and Miss Evelyn Kissin for technical assistance; Dr. Julia Ellis for providing the insulin antiserum used in some of the assays; and the British Diabetic Association for research grants (N.W.O. and R.C.T.).

\section{References}

Albano, J., Ekins., R. P., Maritz, G., and Turner, R. C. (1972). Acta Endocrinologica. In press.

Beard, R. W., Turner, R. C., and Oakley, N. W. (1971). Postgraduate Medical fournal, 47, Jan. Suppl., p. 68.

Blattner, J. R. (1968). Fournal of Pediatrics, 72, 904.

Blattner, J. R. (1968). Fournal of Pediatrics, 72, 904.

Cardell, B. S. (1953). Fournal of Pathology and Bacteriology, 66, 335. fournal of Clinical Investigation, 49, 1517.
Coltart, T. M., Beard, R. W., Turner, R. C., and Oakley, N. W. (1969). British Medical fournal, 4, 17.

Cornblath, M., Joassin, G., Weisskopf, B., and Swiatek, K. R. (1966). Pediatric Clinics of North America, 13, 905.

Cramp, D. G. (1967). Fournal of Clinical Pathology, 20, 910.
Farquhar, J. W. (1956). Archives of Disease in Childhood, 31, 203.

Farquhar, J. W. (1956). Archives of Disease in Childhood, 31, 203. Acta Paediatrica Scandinavica, 58, 481 .

Haworth, J. C., and McRae, K. N. (1965). Canadian Medical Association fournal, 92, 861 .

Oakley, W. G. (1965). In On the Nature and Treatment of Diabetes, ed. B. S. Leibel and G. A. Wrenshall, p. 673. Amsterdam, Excerpta Medica.

Obenshain, S. S., et al. (1970). New England fournal of Medicine, 283, 566.

Paterson, P., Page, D., Taft, P., Phillips, L., and Wood, C. (1968). Fournal of Obstetrics and Gynaecology of the British Commonwealth, 75, 917.

Pedersen, J., and Brandstrup, E. (1956). Lancet, 1, 607.

Sabata, V., Frerichs, H., Wolfe, H. and Stubbe, P. (1970). Fournal of Obstetrics and Gynaecology of the British Commonwealth, 77, 121 .

Saling, E. (1964). Zeitschrift für Geburtshilfe und Gynäkologie, 162, 56.

Seltzer, H. S., Fajans, S. S., and Conn, J. W. (1956). Diabetes, 5, 437.

Turner, R. C., Oakley, N. W., and Nabarro, J. D. N. (1971). British Medical Fournal, $2,132$.

Thomas, K., Gasparo, M. de, and Hoet, J. J. (1967). Diabetologia, 3, 299.

Welborn, T. A., Rubenstein, A. H., Haslam, R., and Fraser, R. (1966). Lancet, 1, 280.

Widdas, W. F. (1961). British Medical Bulletin, 17, 107.

\title{
Syndrome of Rapid Irregular Movements of Eyes and Limbs in Childhood
}

\author{
G. PAMPIGLIONE, MARIA MAIA
}

British Medical fournal, 1972, 1, 469-473

\section{Summary}

Electroencephalographic (E.E.G.), polyelectromyographic (poly-E.M.G.), and electro-oculographic (E.O.G.) studies were made of 16 young children and babies referred because of prolonged episodes of rapid irregular movements of eyes and limbs (R.I.M.E.L.) sometimes involving also the trunk and head. This unusual syndrome of unknown aetiology developed over a period of a few days and persisted for months or years. In the E.E.G. there were only minor changes, without discharges of any kind during the episodes of involuntary movements, and during sleep the spindles were unusually fast. In the poly-E.M.G. single or multiple spikes appeared as electrical concomitants of brief myoclonic phenomena at irregular intervals and independently in the various groups of muscles examined. In the E.O.G. the frequent episodes of irregular jerky movements of the eyeballs occurred at various intervals and without rhythmicity. On clinical observation alone this syndrome may not be easily separated from other conditions such as myoclonic epilepsy, gross ataxia, tremors, or choreiform syndromes in young children; the peculiar combination of neurophysiological findings is essential in the identification of this unusual disorder.

\section{Introduction}

In recent years reports have appeared in the literature of infants affected by involuntary movements of the eyeballs, of the limbs, and sometimes also of the trunk, head, and face. Such cases have been described under a variety of names from

\footnotetext{
Department of Neurophysiology, the Hospital for Sick Children, Great Ormond Street, London W.C.1

G. PAMPIGLIONE, M.D., F.R.C.P., Physician in Charge

MARIA MAIA, M.D., Research Assistant, Honorary Registrar
}

"myoclonic encephalopathy of infants" (Kinsbourne, 1962), "ocular oscillations and truncal myoclonus" (Baringer et al., 1968), "dancing eyes, dancing feet, polymyoclonia" (Dyken and Kolár, 1968) to "infantile polymyoclonia-opsoclonus" (Moe and Nellhaus, 1970). Moreover, some of these unusual motor phenomena have been described in young children with neuroblastoma as "oculo-cerebello-myoclonic syndrome" (Lemerle et al., 1969). However, from the purely clinical descriptions in the literature it is not easy to understand whether the involuntary movements reported under these different names are similar or different phenomena.

The present contribution is based on neurophysiological studies of 16 young children and infants referred to our department because of a syndrome of rapid irregular involuntary movements of the limbs, trunk, head, and eyeballs developing over a period of a few days and persisting for months or years. It seems possible from the neurophysiological findings to identify an unusual clinical syndrome, the aetiology of which is still unknown. We propose in this paper to refer to this syndrome as R.I.M.E.L. (rapid irregular movements of eyes and limbs) referring to the most obvious symptoms and avoiding confusion with other conditions, such as myoclonic epilepsy, ataxia, tremors, and choreo-athetosis.

\section{Patients and Methods}

All the 16 children ( 5 boys and 11 girls) had been admitted to our hospital under the care of one or another member of the staff, but most of them were followed up in the neurological wards. Some clinical features of 6 of the 16 cases in the present paper have been previously reported by Kinsbourne (1962) and these children are included in the present paper with a somewhat different emphasis. The neurophysiological studies included electroencephalography (E.E.G.), polyelectromyography (poly-E.M.G.), and electro-oculography (E.O.G.) with a total of 94 records. Some of the patients were followed up for several years. A cine film was taken of nine children either during or soon after the neurophysiological investigations in order to document some of the odd movements. 
The E.E.G.s were taken with a uniform technique using silver/silver-chloride electrodes stuck to the scalp with collodion according to measurements from bony landmarks (Pampiglione, 1956). The records were taken on Offner Type $D$, or Type $T$, E.E.G. apparatus with an amplification of 10 microvolts per millimetre $(\mu \mathrm{V} / \mathrm{mm})$ pen deflection, a time constant of 0.3 second (occasionally 1 second or D.C.), and a frequency response linear within $10 \%$ up to $70 \mathrm{c} / \mathrm{s}$. The paper speed was usually $3 \mathrm{~cm} / \mathrm{sec}$ (on occasions 1.5 or $6 \mathrm{~cm} / \mathrm{sec}$ ). The polyE.M.G.s were taken also with a uniform technique already described (Pampiglione, 1966) and the paper speed was that used in recording E.E.G.s. The E.O.G.s were recorded from electrodes appropriately placed below and above each eye and at the level of the lateral canthus, the same equipment being used. The time constant was 1 second and the amplification was usually of the order of $30-50 \mu \mathrm{V} / \mathrm{mm}$ pen deflection. Whenever possible the E.O.G. the E.E.G.s, and the poly-E.M.G. were recorded together in order to demonstrate the coincidence, or lack of coincidence, in the occurrence of the involuntary movements and any change in the E.E.G. features (see Figs 1-3).

Various stimuli were presented either in the form of flashes of light or sudden noises, while on other occasions tactile stimuli were used. Sometimes a toy was presented to the child or removed from his hand to study the changes induced in his clinical state and documented by the poly-E.M.G., E.O.G., and E.E.G. Sleep was obtained spontaneously in eight children and was induced in a further six with quinalbarbitone.

In all children chest and skull $x$-ray films, blood counts, fasting blood sugar, and plasma electrolyte studies failed to show any abnormality. Amino-acid pattern was found to be normal in the urine of the eight children tested and in the plasma of the five children tested. In the seven cases from whom cerebrospinal fluid samples were taken there were no consistent abnormalities. Virological studies and antibody titres did not suggest the presence of an active infection.

Lumbar air encephalography was performed on five children, with normal findings in two, "mild cerebral atrophy" in two others, and a slight ventricular asymmetry in the fifth. Air ventriculography was normal in all three cases in which it was performed.

Histological studies were carried out in one case from a brain biopsy specimen (Professor Blackwood) and in another from postmortem material (Dr. Trevor Hughes). However, in neither case could any distinctive pathological condition be found in the brain, and only minor changes of doubtful significance were seen.

\section{Results}

The disease started between the ages of 4 and 18 months in 13 cases while in the remaining three it began between 21 and 30 months. The initial complaints were vomiting in six cases, unsteadiness in three, shaking of the limbs in three, odd movements of the eyes and/or head in three, while an increased startle response to unexpected noises had been reported in the last case. The condition evolved rapidly and usually the full syndrome was established within three days, except in two patients in whom "bouts of shaking of the limbs" had been the only features for a period of two and eight months respectively. Restlessness, irritability, and loss of motor skills were prominent features in all children, who became grossly incapacitated by the involuntary movements, usually within a very few days.

The course of the disease showed considerable individual variations: in two recent cases the period of clinical follow-up was too short to assess the long-term outcome. In three patients the disorder appeared as a single episode although prolonged for months. In five children several episodes had occurred over a period of a few years, each lasting some weeks or a few months with complete freedom between these episodes. In the remaining six children the disease continued for some years, often fluctuating in severity but without any symptom-free period.
In all patients there was, during the more obvious phase of the disease, irregular jerking of the limbs, eyeballs, and often trunk, usually facilitated by unexpected stimuli, and commonly, though not always, disappearing during sleep. The irregular movements of the eyeballs and those of the limbs or trunk did not occur simultaneously (see Figs. 1-3). The neurophysiological investigations were carried out at various stages of R.I.M.E.L.

The E.E.G. showed a fairly uniform pattern in all patients with fairly well-preserved rhythmic activity for the age although sometimes there was a moderate excess of generalized irregular intermediate slow activity and or faster components. No gross asymmetries were found between the activities of the two hemispheres and on no occasion did spikes or complex wave forms appear in either the waking state or sleep. In particular, no discharges of any kind were seen either during the periods of relative quiescence of the condition or during the bouts in which the involuntary movements were gross. The responses to photic stimulation were well preserved and no discharges were elicited. During sleep (whether spontaneous or induced) runs of $16 \mathrm{c} / \mathrm{s}$ waves appeared in the frontocentral regions instead of the more usual 12-14 c/s sleep spindles. Sudden noises during sleep evoked fairly symmetrical K-complexes. During the waking state passive eye closure elicited the appearance of rhythmic activity in the occipital region on occasions, but in general this manoeuvre made the child extremely irritable, with a pronounced increase in involuntary movements often involving the head and particularly the eyeballs as well as the trunk and limbs.

In the poly-E.M.G. the features were similar in all cases; the "involuntary" activity of each group of muscles occurred independently. Such muscle activity appeared in the form of either single or multiple spikes which kept on reappearing somewhat erratically without any periodicity and, in particular, without the rhythmicity which is characteristic of a tremor. During a voluntary action, such as attempting to hold a toy, there was often some irregular discontinuity in the grouping of the muscle action potentials in the agonist muscles. Such discontinuity was not obvious in the antagonist group of muscles. Moreover, there was none of the "spreading activity" seen in children with spasticity during a voluntary movement (Pampiglione, 1966). Stretch reflexes were usually diminished rather than increased, and were sometimes absent. No clonus could be elicited. When the child was upset the involuntary movements tended to increase, and sometimes wild irregular jerking of the whole body and limbs appeared without rhythmic phenomena. During photic stimulation if the child was not frightened there was a diminution rather than an increase in the involuntary movements, particularly if the child became interested in the flickering light.

During a voluntary movement, instead of a continuous activity in the E.M.G. there was a considerable irregularity and discontinuity; both the grouping of the muscle action potentials and the periods of relative silence were of short duration-that is, of the order of 20-50 milliseconds. When E.E.G., polyE.M.G., and E.O.G. were recorded simultaneously there was no obvious E.E.G. change during even the wildest bouts of involuntary movements, and in particular no spikes or complex wave forms ever appeared in the E.E.G.

In the E.O.G. the variations in differences of potential (related to the displacement of the eyeballs, either in the vertical or in the horizontal plane) were erratic with variable direction and speed. Most of the wild movements of the eyeballs tended to occur in bouts, usually symmetrically in the two eyes whatever the amplitude of the excursion. Rhythmic phenomena such as those seen in nystagmus were usually absent, but on occasions, during some more prolonged bouts, a brief run of less irregular rapid components occurred at about 5-12 c/s. In one child with this syndrome a monocular pendular nystagmus was seen and recorded in addition to the bilateral irregular jerky movements of the eyeballs.

The main neurophysiological features of this peculiar syndrome are illustrated by the following cases. 

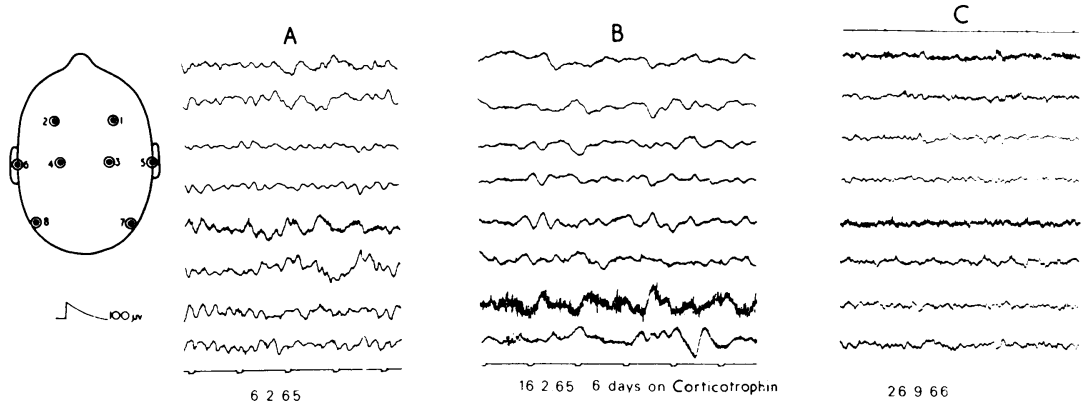

FIG. 1-Case 1. (A) E.E.G. taken at age of 15 months; (B) during treatment with corticotrophin for six days; (C) at age of 2 years and 11 months. The E.E.G. montage is indicated on the head diagram (average reference). Increase in irregular slow activity in B during corticotrophin treatment (time scale in seconds) In $D$ and $E$ the first two two channels record the E.E. the right central, vertex, and left central region. Channels 3 and 4 record vertical movements of right and left eye. Channels 5 and 6 record horizontal movements of both eyes (respectively from electrodes near the lateral canthus and below the eyes). Channels 7 and 8 record the E.M.G. from right biceps and right triceps. The bouts of involuntary movements of the eyeballs appear independently in the vertical and horizontal directions pend are not accompanied by any discharges in E.E.G. or by gross alterations in the E.M.G.
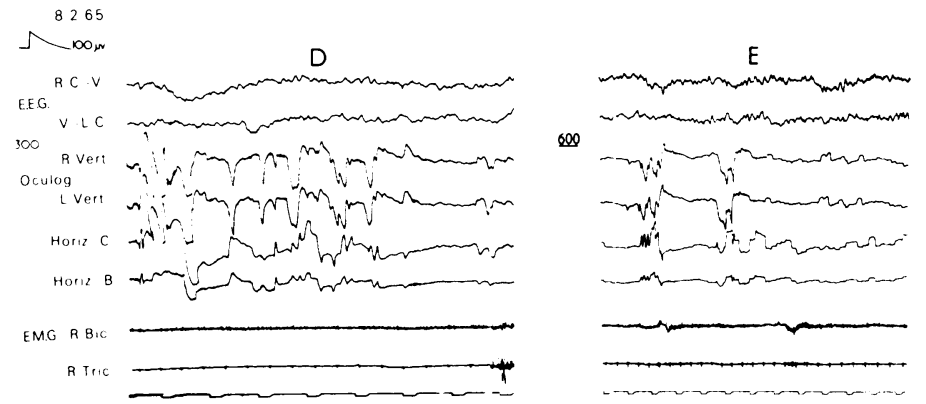

\section{Case 1}

This child was under the care of Dr. Macdonald Critchley when admitted at the age of 15 months in February 1965. The symptoms began at the age of 13 months, and she was referred to the department of neurophysiology as a possible case of encephalitis. A number of E.E.G.s were taken over a period of two years; in the acute phase of the disease, at the age of 15 months, the rhythmic activity in the E.E.G. though somewhat slower than normal appeared well preserved. There was some excess of slow components slightly more obvious over the posterior than over the anterior half of the two hemispheres but there were no lateralizing signs and no paroxysmal features. During treatment with corticotrophin the E.E.G. showed a considerable increase in slow waves of the order of $100 \mu \mathrm{V}$ at about $1-4 \mathrm{c} / \mathrm{s}$ over both hemispheres with a diminution in the normal rhythmic activity six days after the beginning of corticotrophin treatment (a fairly common cerebral response to this drug). The excess of slow activity disappeared after discontinuation of corticotrophin. On follow-up there was some normal maturational progress in the electrical activity of the brain and no discharges were seen at any time (Fig. 1, A, B, and C).

A combined E.E.G., poly-E.M.G., and E.O.G. during the active phase of the disease and before the beginning of corticotrophin treatment is shown in Fig. 1 (D and E). The usually irregular eye movements with occasional bouts of less dysrhythmic oscillations appeared independently in the vertical and horizontal planes of each eye. The involuntary movements of the limbs (recorded by polyE.M.G.) were infrequent and did not coincide with the rather prominent, wild eye movements.

This girl was given corticotrophin for about a month and the abnormal eye movements stopped in less than one week. She began to walk again at 2 years but she made slow progress in motor development and was admitted again at 3 years. However, later she developed normally, and at 5 years she started school, and at 7 years a psychometric assessment gave an I.Q. of 105. She was doing well at school. No relapse occurred.

\section{Case 2}

This boy was originally thought to have a posterior fossa tumour because of a peculiar jerkiness of the arms noticed from the age of 11 months. He was admitted at the age of 20 months to the neurosurgical unit under the care of Mr. K. Till on 6 October 1964. An E.C.G. taken on 8 October, during an acute phase of the disease, showed a moderate excess of irregular intermediate slow activity and a fair amount of rhythmic activity appropriate to the child's age (features not commonly seen in posterior fossa tumours). Burr-hole ventriculography on 12 October showed only a mild dilation of the ventricular system. Dr. K. Zilkha (23 October) suspected a small brain stem lesion, and the late Dr. Paul Sandifer ( 28 October) thought this boy might have acute cerebellar ataxia. On corticotrophin the involuntary movements disappeared in about one month, although the boy remained unsteady. A relapse occurred in February 1965 and he was readmitted under the care of Dr. Macdonald Critchley. Spontaneous remission occurred in a few weeks on no drugs. The irregular eye movements illustrated in Fig. 2 were neither preceded nor accompanied by detectable E.E.G.

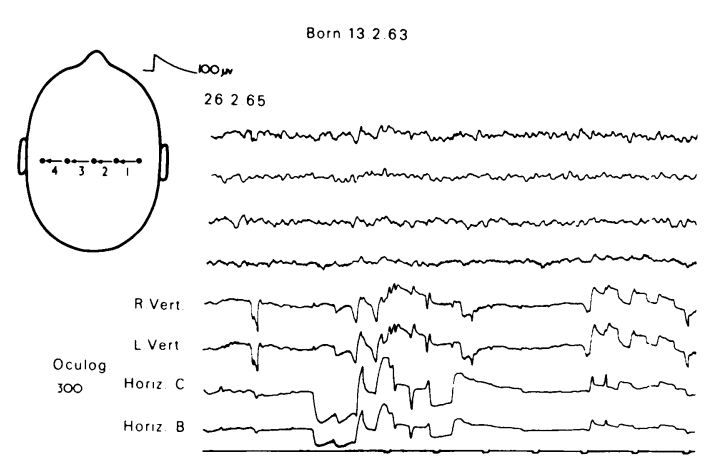

FIG. 2-Case 2. Record taken at time of relapse in February 1965. The first four channels record the E.E.G. from the regions indicated in the head diagram. The remaining four channels record the vertical and horizontal movements of the eyes. Note the independence of vertical and horizontal rapid irregular movements of the eyes without any concomitant change in the E.E.G.

changes and were prominent in the vertical plane, although others also occurred in the horizontal plane quite independently.

This child remained ataxic for a further period of nearly two years but eventually even the ataxia disappeared. He attended a school for the educationally subnormal, with an I.Q. of 74 (June $1969)$ at the age of nearly $6 \frac{1}{2}$ years.

\section{Case 3}

This boy had originally been admitted at the age of 9 months under the care of Dr. R. Lightwood but was also seen over the years by several other physicians, including Dr. Sandifer, Sir 
Wilfred Sheldon, Dr. K. Zilkha, and Professor Stroud. He was believed to be suffering from myoclonic epilepsy and was also seen in private consultation by Dr. Ludo Van Bogaert, who thought he might have an unusual form of brain stem encephalitis. This is Case No. 3 of the group clinically described by Kinsbourne (1962).

In the E.E.G.s taken at the ages of 9 and 10 months during the waking state and during sleep (Fig. 3) there were no paroxysmal features and there was some preservation of normal rhythmic
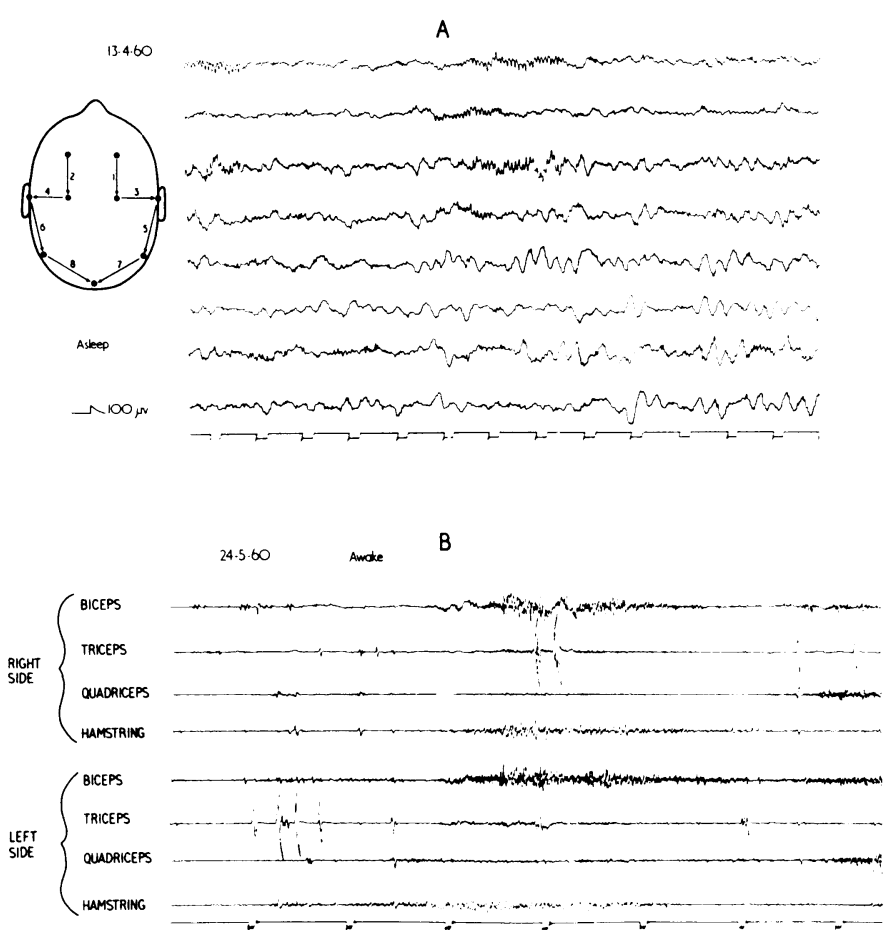
Fig. 3-Case 3. (A) E.E.G. during sleep when the "spindles" were at $16 \mathrm{c} / \mathrm{s}$
instead of the normal 12-15 c/s (13 April 1960). Paper speed is $3 \mathrm{~cm} / \mathrm{sec}$. instead of the normal 12-15 c/s (13 April 1960). Paper speed is $3 \mathrm{~cm} / \mathrm{sec}$. (B) Poly-E.M.G. during the waking state from biceps, triceps, quadriceps,
and hamstring group of muscles of right and left arm and leg. Isolated large or small spikes accompanying a jerk of the limb appear independently in the various muscles examined though maximal in the triceps (24 May 1960). Paper speed is at $6 \mathrm{~cm} / \mathrm{sec}$.

activity. During sleep most of the E.E.G. features were within normal limits, but instead of the usual sleep spindles at $12-14 \mathrm{c} / \mathrm{s}$, runs of $16 \mathrm{c} / \mathrm{s}$ waves were seen with variable lateralization in the frontocentral regions. In the poly-E.M.G. the muscle action potentials appeared of large amplitude as single spikes in the triceps in particular, without either simultaneous or "reflex" activity in the antagonist group of muscles (Fig. 3).

Several courses of corticotrophin were given, usually with some temporary benefit. Eventually the involuntary movements disappeared and the child made reasonable progress at school. He has had no recurrence of involuntary movements for over six years.

\section{Effect of Treatment}

The response to corticotrophin or steroids has been rather variable both clinically and neurophysiologically in this group of children, but some of them certainly improved over a period of a few weeks, three of them showing improvement within three to four days after the beginning of corticotrophin treatment. However, in a disorder of obscure aetiology such as R.I.M.E.L., with spontaneous fluctuations in symptomatology and occasional spontaneous relapses, it was difficult to assess the effect of treatment. The outcome was reasonably uniform with diminution or disappearance of the involuntary movements regardless of the amount of corticotrophin given. In the four patients who did not have corticotrophin the outcome was similar. Some degree of mental retardation was found at follow-up in six of the 16 patients whether or not treated with corticotrophin.

\section{Discussion}

"Irregular twitches of one or the other part of the body in multimuscular groups" were described by Russell Reynolds over 100 years ago in patients with myoclonic epilepsy, and this form was often colloquially known as "the jumps" (Wilson, 1940). The clinical term "myoclonic jerks" seems to cover a wide range of involuntary movements although it implies phenomena of short duration. However, in an extensive review of the literature on myoclonus, Halliday (1967) included any muscular contraction lasting from a few milliseconds to a second or perhaps more, such as the complex movements of a repetitive kind seen in patients with subacute sclerosing panencephalitis, in contrast with previous studies on this condition (Pampiglione, 1964).

The literature on myoclonic phenomena is very extensive but each author expresses his own views, often without measurable identification of the involuntary movements. In a review of myoclonic syndromes Gastaut (1968) attempted to put some order into the electroclinical classification of these involuntary movements, and limited the duration of myoclonus to 20-60 milliseconds as measured from the E.M.G. This seems a very useful working criterion, with which we agree. The children described in the present paper showed mostly short-duration phenomena in the poly-E.M.G. during the irregular jerking of the limbs.

Our material includes the six cases described by Kinsbourne (1962), but his claims that "the diagnosis can be made with confidence on clinical grounds alone" is not supported by our larger series of cases. In fact, the provisional clinical impressions formulated before the neurophysiological investigations in our 16 cases were as follows: (a) cerebellar ataxia or degeneration ( 5 cases), (b) posterior fossa tumour ( 3 cases), (c) "dancing eye syndrome" ( 3 cases), ( $d$ ) encephalitis ( 3 cases), and (e) myoclonic epilepsy ( 2 cases).

A number of other cases had been referred by experienced clinicians for neurophysiological investigations with a provisional diagnosis of "dancing eye syndrome," but most of these children showed spikes or complex wave forms in the E.E.G., of a type commonly seen in patients with myoclonic epilepsy, who improve on anticonvulsants in contrast with children suffering from R.I.M.E.L. Other patients who were known epileptics on drugs showed in their poly-E.M.G. the rhythmic phenomena typical of a tremor possibly of toxic origin which improved after reduction of anticonvulsant dosage.

Another child of $2 \frac{1}{2}$ years was referred for neurophysiological investigations by Dr. J. Wilson as a case of neuroblastoma with the "dancing eye syndrome." This case was not included in our series because when this child was seen two days later in our department no involuntary movements of any kind could be detected, even after various manoeuvres which usually facilitate jerks in R.I.M.E.L. Without neurophysiological documentation it was not possible to assess whether the involuntary movements seen a few days earlier might have been some form of tremor or an exceptionally brief period of R.I.M.E.L.

Dyken and Kolár (1968) suggested that some immunological factor may be relevant to the aetiology of "dancing eyes, dancing feet, infantile polymyoclonia." The association of neuroblastoma with the "infantile polymyoclonia-opsoclonus syndrome" has been reviewed by Moe and Nellhaus (1970). However, in our 16 cases of R.I.M.E.L.no evidence of neuroblastoma emerged at follow-up from a minimum of three months to a maximum of 12 years (average six years) from the beginning of symptomatology.

Moreover, if we agree that R.I.M.E.L. may be defined by its electroclinical criteria, all the cases of neuroblastoma with alleged "polymyoclonia" reported in the literature have had little if any electrophysiological documentation of the involuntary movements. In some papers "tremor" is often mentioned and this presupposes a rhythmic (rather than irregular) phenomenon with a precise relation between the activities of agonist and antagonist muscles quite different from our findings in 
R.I.M.E.L. In the illustration by Moe and Nellhaus (1970) the ocular movements recorded seem slow rather than rapid and seem to be independent in each eye (an unusual phenomenon in any disease); moreover the E.M.G. shows rhythmic grouping of muscle action potentials.

In any consideration of involuntary movements, whether at limb or eye level, the electrographic features represent a permanent record of the abnormal motor phenomena. In the differential diagnosis of R.I.M.E.L. the E.E.G. and E.O.G. features are essential physical signs, which differ from the findings in myoclonic epilepsy, the cerebellar ataxias, tremors, and the choreiform syndromes even if the aetiological factors remain at present obscure.

We are indebted to our colleagues at the Hospital for Sick Children and in particular to Drs. E. Brett, Macdonald Critchley, the late Paul Sandifer, and J. Wilson. Support from Action for the Crippled Child is gratefully acknowledged.

\section{References}

Baringer, J. R., Sweeney, V. P., and Winkler, G. F. (1968). Brain, 91, 473. Dyken, P., and Kolár, O. (1968). Brain, 91, 305.

Gastaut, in (1968). Revue Neurologique, 119, 1.

Halliday, A. M. (1967). Brain, 90, 241.

Kinsbourne, M. (1962). Fournal of Neurology, Neurosurgery and Psychiatry, 25,271 .

Lemerle, J., Lemerle, M., Aicardi, J., Messica, C., and Schweisguth, O. (1969). Archives Françaises de Pédiatrie, 26, 547

Moe, P. G., and Nellhaus, G. (1970). Neurology, 8, 756.

Pampiglione, G. (1956). Proceedings of the Electrophysiological Technologists Association, $7,20$.

Pampiglione, G. (1964). Archives of Disease in Childhood, 39, 558.

Pampiglione, G. (1966). Fournal of the Neurological Sciences, 3, 525.

Wilson, S. A. K. (1940). Neurology. London, Arnold.

\title{
False-negative Results in Brain Scanning
}

\author{
E. H. BURROWS
}

British Medical fournal, 1972, 1, 473-476

\section{Summary}

There were 118 false-negative results in a series of 847 cases of proved intracranial lesions subjected to brain scanning. In patients with neoplasms false-negative results are much more likely if the site of the tumour is infratentorial or mediobasal. In patients with stroke the incidence of false-negative results depends on the stroke-scan interval.

\section{Introduction}

The value of a technique lies in a knowledge of its limitations, and this is particularly true in brain scanning, which is accepted by many as a blanket test of intracranial lesions. Certain intracranial lesions may give false-negative results, and a knowledge of the pattern is important clinically in the choice of appropriate diagnostic procedures.

In this paper a review is presented of the false-negative brain scans in a series of 847 patients with proved intracranial lesions, all of whom had undergone cerebral scintigraphic examination in the course of other investigations and treatment in the Wessex Neurological Centre, Southampton.

\section{Patients and Methods}

A survey was made of the final clinical diagnosis of about 2,000 patients subjected to brain scanning, and 847 were found in whom intracranial lesions had been proved to be present. The cerebral scintigrams of these patients were then reviewed and the results studied in relation to the size, site, and nature of the intracranial lesions. Care was taken to ensure that there was no reasonable doubt about the final diagnosis, though histological proof was not always available. For example, angiographic tumour staining followed by clinical deterioration and death was accepted evidence of a glioma or, in the presence of a

Wessex Neurological Centre, Southampton

E. H. BURROWS, M.RAD., D.M.R.D., Consultant Neuroradiologist primary cancer elsewhere, metastatic malignancy. Clinical recovery from a stroke with a positive-to-negative brain scan sequence was labelled as a cerebrovascular accident; a diagnosis of intracerebral haematoma was made only if blood was demonstrated by lumbar puncture or burr-hole biopsy.

All the patients were examined with a rectilinear scanner (Magna Scanner V, Picker) and 52 with a gamma camera (Pho-Gamma, Nuclear-Chicago) as well. The examinations were performed as carefully as possible, in order to eliminate technical causes of failure. The radiopharmaceuticals used were ${ }^{197} \mathrm{Hg}$-chlormerodrin, ${ }^{99} \mathrm{~m} \mathrm{Tc}$-pertechnetate, and ${ }^{113 \mathrm{~m} I n-}$ DTPA. In addition to radioindium scanning, 108 patients were double-scanned with one of the other substances as well. Computer aids were not used, either in obtaining or interpreting the results.

\section{Findings}

Of the 847 proved intracranial lesions, 118 (13.9\%) showed no abnormal uptake on brain scanning-that is, false-negative results. The latter were distributed as follows: 616 neoplasms, $74(12.0 \%) ; 211$ vascular lesions, $44(20.9 \%) ; 20$ inflammatory lesions, 0 . The incidence in individual lesions is given in Table $\mathrm{I}$, and their topographical distribution in Table II. These results are analysed in detail.

TABLE I-Brain Scanning in 847 Proved Intracranial Lesions: Histology of 118 False-negative Lesions

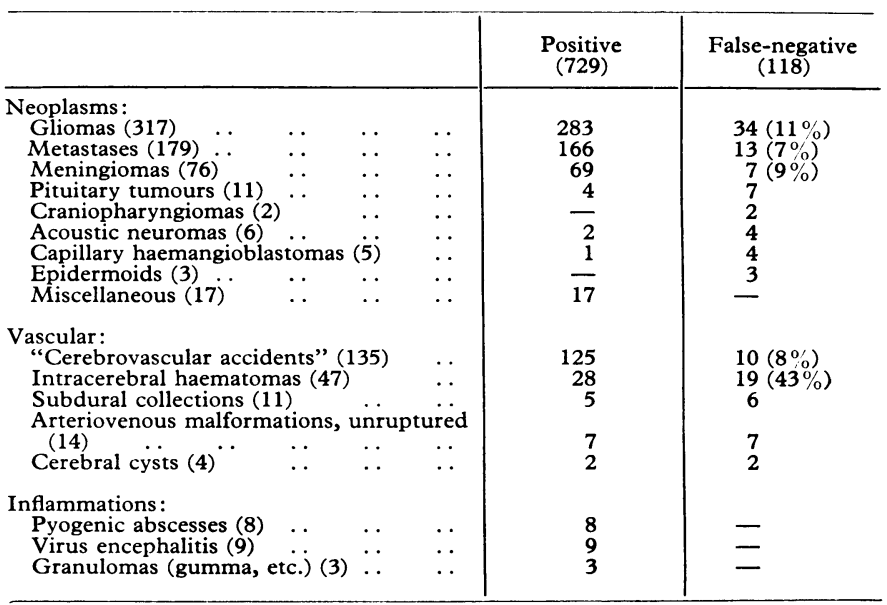

